\title{
Evaluation of thermally stable phosphor screens for application in Laser diode excited high brightness white light modules
}

\author{
A.Salimian ${ }^{1}$, J. Silver ${ }^{1}$, G.R. Fern ${ }^{1}$, M. Evans ${ }^{1}$ and R. Haghpanahan ${ }^{2}$ \\ 1. Centre for Phosphors and Display Materials, Wolfson Centre for Materials Processing, Institute for Materials and manufacturing, Brunel \\ University London, Kingston Lane, Uxbridge, Middlesex, UB8 3PH, United Kingdom \\ 2. Department of Electronics and computer engineering, Brunel University London, \\ Kingston Lane, Uxbridge, Middlesex, UB8 3PH, United Kingdom
}

A study on the preparation of thermally stable phosphor targets based on yttrium aluminium garnet doped with cerium (YAG:Ce) when excited by a high power laser diode is described. The luminous flux, chromaticity and Radial spectral flux of the targets along with their thermal stability have been determined when exposed to laser powers of up to $5000 \mathrm{~mW}$. This report presents successful high brightness light sources with adjustable emission properties achieved by utilising thermally stable phosphor targets excited by high power laser diodes.

\section{Introduction}

With advances in the manufacture and development of blue laser diodes (BLDs), various commercial and research areas are opening toward BLD applications. ${ }^{1}$ These applications include areas such as projection displays, automobile headlamps and illumination systems requiring white light source with high brightness output. The BLD laser beam can be used to activate a phosphor material to generate high brightness emission with photometric properties which relate to both the phosphor material being used and the emission wavelength of the laser. The concept of creating a white light emission based on BLD laser phosphor interaction was demonstrated initially by Nichia. ${ }^{2}$ This white light source was named "Micro white" due to the small emission spot size of the light source. ${ }^{3}$

In the last seven years two early types of laser diodes (LDs) based white light sources were introduced; using 405nm violet LDs and 450nm BLDs. ${ }^{4,5}$ The mechanism of light emission is simply very similar to the application of phosphors in light emitting diodes (LEDs). The laser beam from the diode activates the phosphor material depending on the beam's wavelength and band gap of the phosphor material which will result in emission of light with wavelengths dependent on the nature of the phosphor alone or combined with some light (not converted from the laser source). The combination of the emitted light (from the phosphor) mixed with the scattered laser light produces an overall emission spectrum with characteristics which can be manipulated by altering the amount of and nature of the emitting phosphor. For example, a BLD activating a yellow emitting phosphor will result in the mixing of the yellow and blue light and hence a white light will be generated. One important factor that needs to be considered when choosing the laser pump source and the phosphor material is that the Stoke's shift loss at the phosphor increases as the wavelength difference between the exciting light (Laser) and the emission light from the phosphor increases. The photometric and colorimetric properties of a BLD (445nm) activating an InGaN phosphor has been reported by Ryu. ${ }^{3}$ They investigated the effects of the BLDs current and voltage and temperature on the emission characteristics of the phosphor however they did not report on the structure of the phosphor target and means of thermal management at the phosphor level which will directly affect the efficiency of the conversion. The temperature of the phosphor target upon exposure to the laser beam will elevate rapidly and this severely affects the emission from the phosphor since at higher temperatures the performance of the phosphors drops of in some cases dramatically.

In this report we will describe the photometric properties of the garnet structured YAG:Ce phosphor emitting under stimulation from a 450nm BLD pump with emphasis on the structuring of the phosphor target to maintain efficient thermal dissipation at the phosphor target level. 


\section{Experimental}

Materials. -Four types of phosphor target systems were prepared using a commercial grade YAG:Ce phosphor. All four target systems (A, B, C \& control) contained the same phosphor material; however $\mathrm{A}, \mathrm{B}$ and $\mathrm{C}$ were structured so that each had a different method of thermal dissipation in place, whereas the control had no thermal dissipation in place, the laser pump was constructed from three Osram PL TB450B 1.6W laser diodes in a TO56 package and assembled in a copper heat sink. A more powerful $5 \mathrm{~W}$ laser module was constructed by using a Nichia diode NDB7A75. The targets were constructed as follows:

System A: Powder phosphor material was speed mixed with relevant additive (A) at $20 \mathrm{~K} \mathrm{rpm}$ for 1 minute so that the mixture contained a certain percentage of phosphor to A by mass. The target was then placed in an aluminium block housing using a thermal paste adhesive to ensure the thermal energy could dissipate into the aluminium heat sink.

System B: Powder phosphor material was speed mixed with relevant additive (B) at $20 \mathrm{~K} \mathrm{rpm}$ for 1 minute so that the mixture contained a certain percentage of phosphor to B by mass. The target was then placed in an aluminium block housing using a thermal paste adhesive to ensure the thermal energy could dissipate into the aluminium heat sink.

System C: Powder phosphor material was speed mixed with relevant additive (C) at $20 \mathrm{~K}$ rpm for 1 minute so that the mixture contained a certain percentage of phosphor to $\mathrm{C}$ by mass. The target was then placed in an aluminium block housing using a thermal paste adhesive to ensure the thermal energy could dissipate into the aluminium heat sink.

Control: Powder phosphor material was used on its own and embedded in a solid matrix without significant thermal dissipation properties. The target was then placed in an aluminium block housing using a thermal paste adhesive to ensure the thermal energy could dissipate into the aluminium heat sink.

Measuring methods.-To obtain photometric characteristics of the samples they were placed in an integrating sphere. The sphere was calibrated so that the absorption of light by the phosphor target body assembly was accounted for during the analysis. The samples were then activated using a laser source which was installed on the outside body of the sphere with the beam directly focused on the phosphor target. The sphere was coupled to a spectrometer to obtain photometric and colorimetric measurements. The geometry used for the control, A and B targets is presented in figure 1. The laser source was constructed so that the power output of the laser could be adjusted between $0.5 \mathrm{~W}$ to $3 \mathrm{~W}$ depending on the voltage supplied to the BLD. The surface temperature of the target once exposed to the laser beam was monitored so that the thermal dissipation method used could be evaluated. 

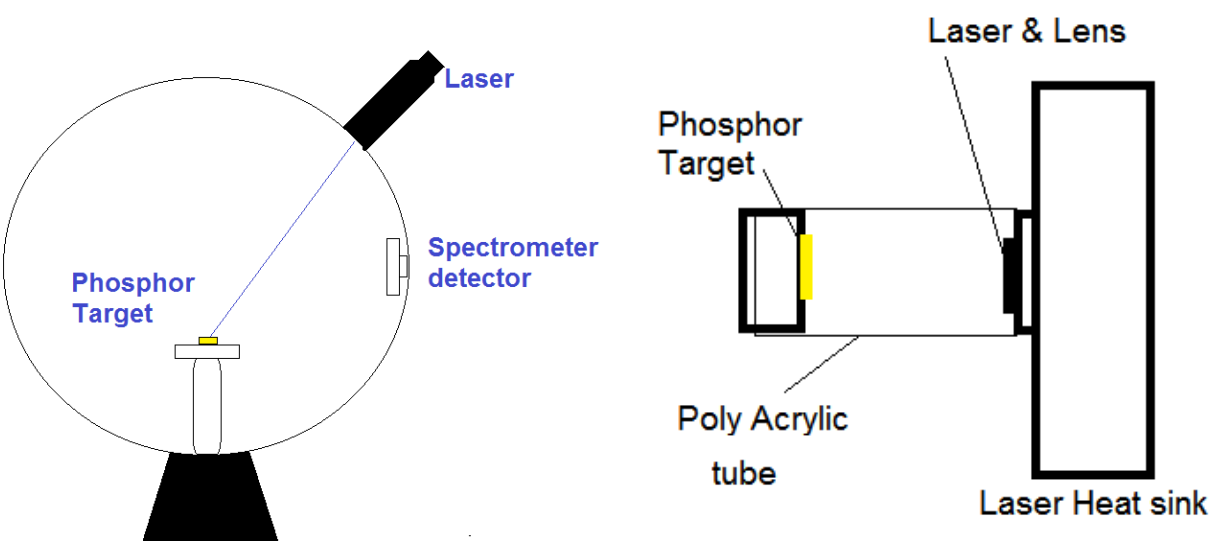

Figure 1. The experimental set up used showing how the emission characteristics of the assembled phosphor target were investigated using an integrating sphere coupled to a BLD and a spectrometer. , Left: used as is for the control, A \& B targets. Right: The structure of the module that was used as the $\mathrm{C}$ target that was then positioned in the same target position.

For target $\mathrm{C}$ the set up was different. A $5 \mathrm{~W}$ constant beam module was designed so that the whole module could be placed inside the sphere. The module was designed by packaging the laser and the $\mathrm{C}$ target system into one body that was wrapped in a poly acrylic tube which was used as a window for the emission the light. For this $\mathrm{C}$ target the laser was inside the sphere at the point the other targets were sited. The structure of the module with target $\mathrm{C}$ is presented in figure 1 (right).

\section{Results and Discussion}

The thermal management of the phosphor target is very important in applications where BLDs are used to activate phosphor light source structures so that the efficiency of the phosphor is not compromised. Increase in temperature directly affects the efficiency of the phosphor and without dissipation of the heat, light output will significantly decrease. To demonstrate the effect of heat in the sample an experiment was carried out where the control sample was exposed to the beam. It was exposed to $1.5 \mathrm{~W}$ of $450 \mathrm{~nm}$ blue laser light. The luminous flux, spectral flux \& chromaticity of the output emission of the sample over a period of 1 second with 0.1 second intervals were obtained. The results obtained from this experiment are presented in figure 2 where the drop in efficiency and reduction of brightness in lumens is extremely rapid (less than 0.7 seconds). As described the reduction of light output is related to a drop in phosphor efficiency and it is further elaborated in figure 3 which demonstrates the change in chromaticity index of the light output over a period of one second. The temperature of the target was monitored with a FLIR thermal camera to measure the rise in temperature of the phosphor target without thermal management which is presented in figure 4. 

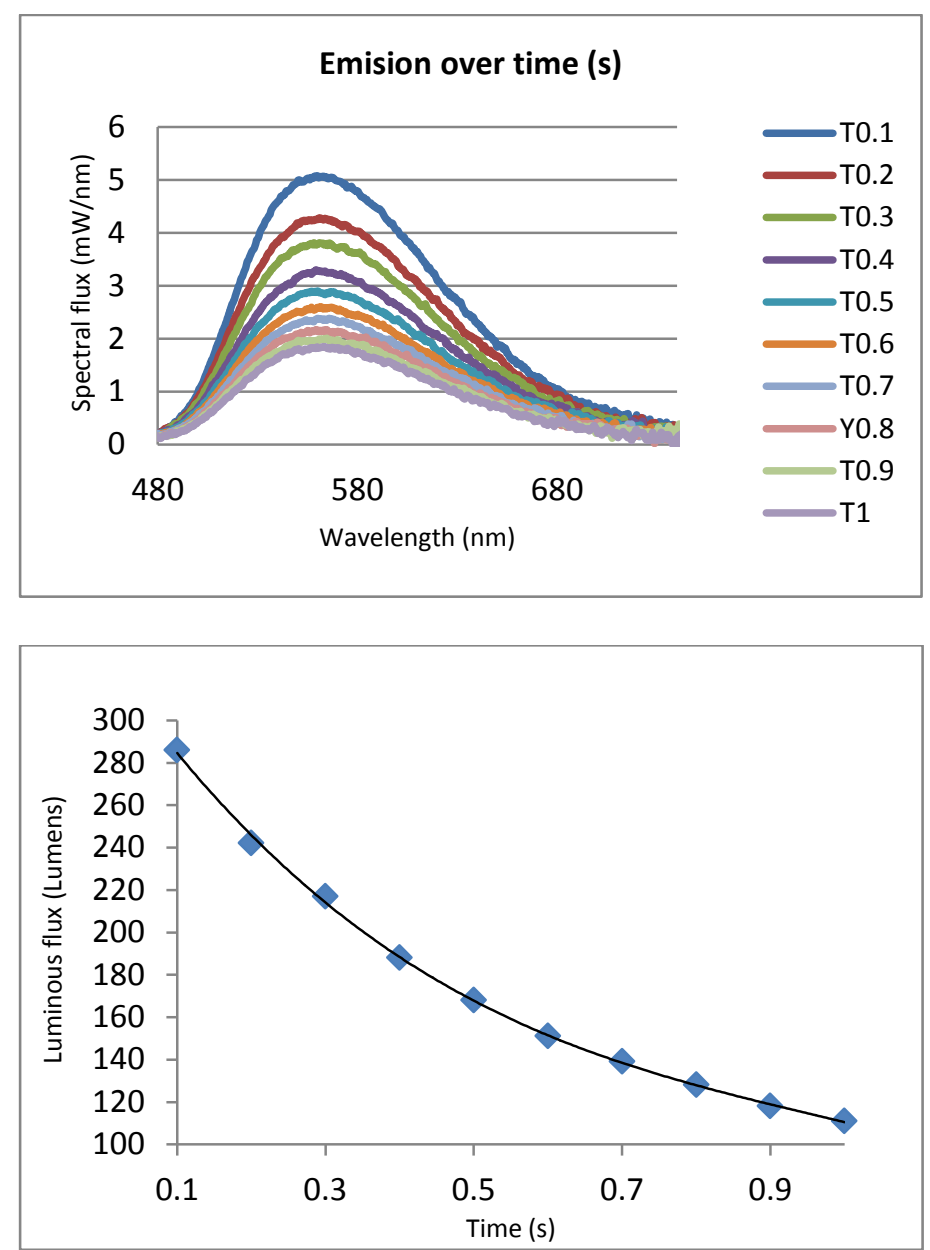

Figure 2. Drop in emission over a period of 1 second without thermal management of the control target. 


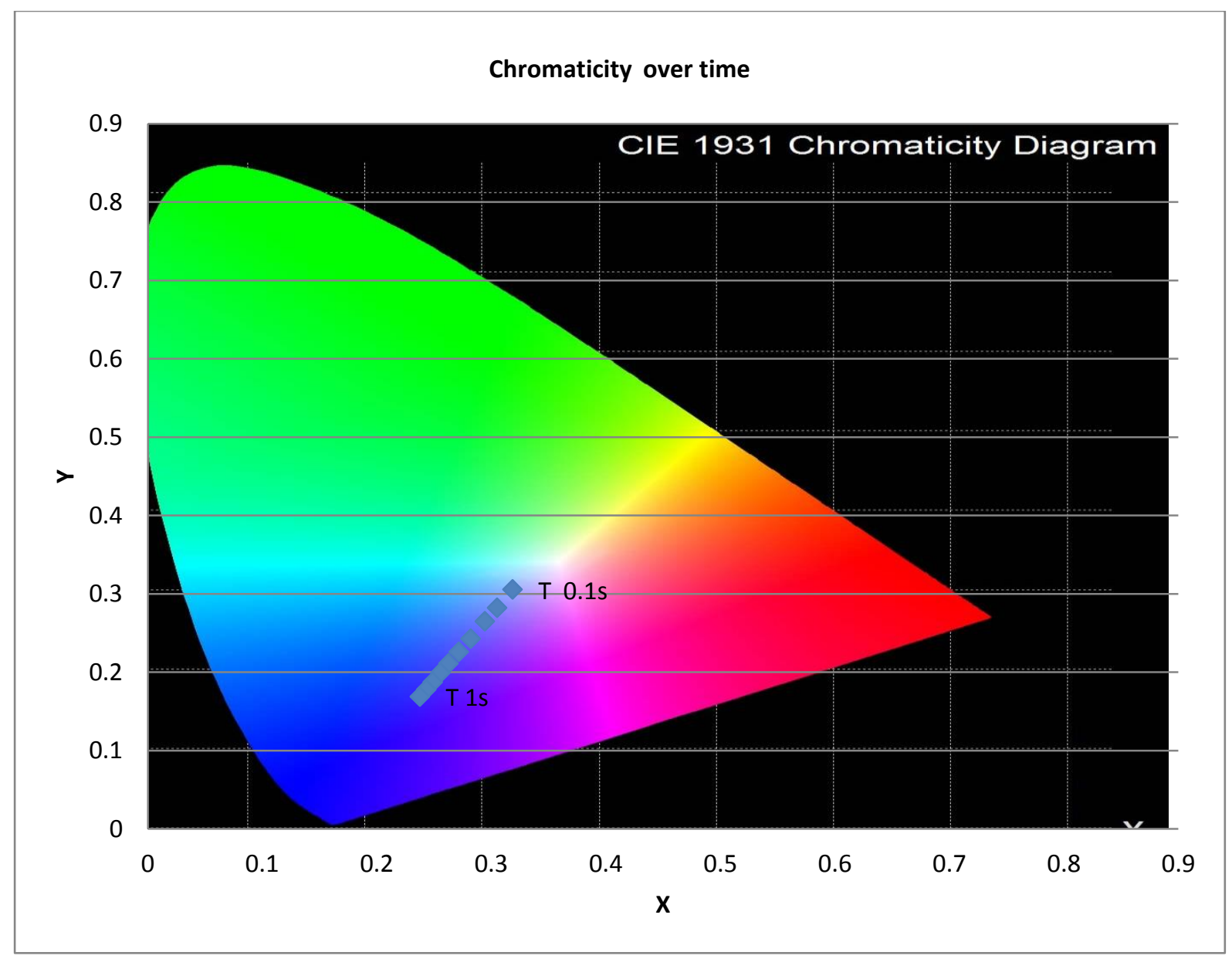

Figure 3. The Variation of the Chromaticity coordinates of the Control phosphor target exposed to a $1.5 \mathrm{~W}$ laser power over a period of one second. The colour of the emission shifts toward blue (colour of the laser) as the phosphor is heated by the laser.

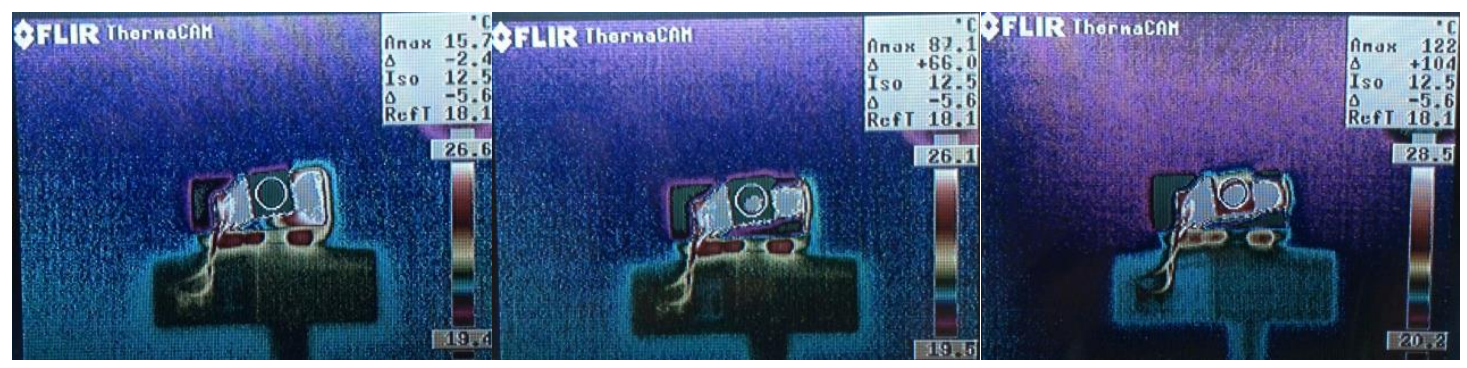

Figure 4. The temperature of phosphor target without thermal management is increased from 15 degrees Celsius (left) to 87.1 degrees within 1 second (centre) and reaches up $122^{\circ} \mathrm{C}$ (right) in just 15 seconds. 
The A target was then studied in exactly the same way as the control and the emission characteristics of the target were monitored under various BLD laser powers where the laser energy varied from $0.5 \mathrm{~W}$ to $3 \mathrm{~W}$. To eliminate the thermal effect, each reading was taken separately to ensure that the sample was not heated, the temperature of the target was monitored as described earlier. There is a linear relationship between the laser power and the luminous flux of the target. Theoretically there is a point where the phosphor will be saturated, however in our experiments the maximum available laser power that could be focussed on one point was $3 \mathrm{~W}$ and up to this point, a single spot of $2 \mathrm{~mm} \times 5 \mathrm{~mm}$ was evaluated and no saturation was observed. However this illustrates that the light output can be formulated with respect to the laser power that is directed at the target prior to a saturation point. The results are presented in figure 5.

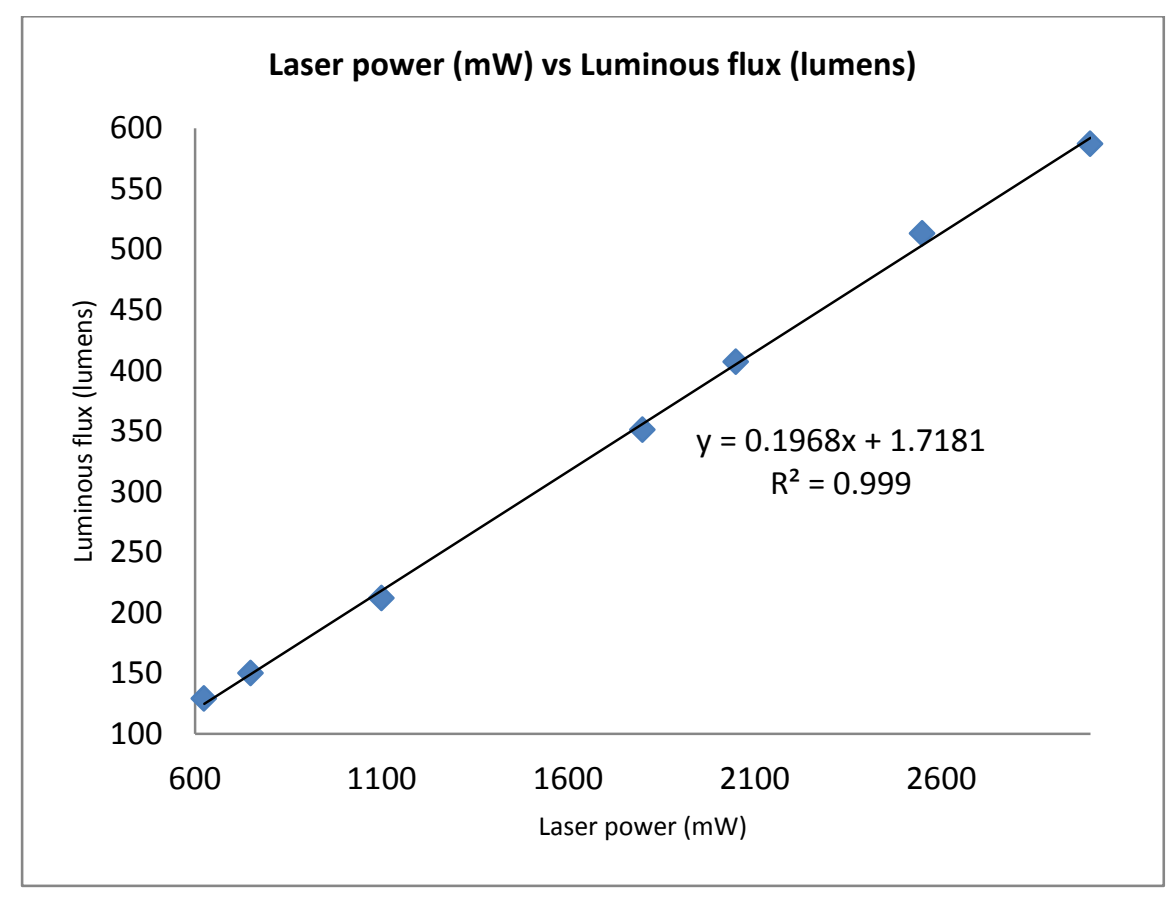

Figure 5. Relationship between laser power and Luminous flux of the thermally managed target A.

A set of phosphor type A targets were made. The targets were prepared as described earlier with each target containing different amount of phosphor to heat transfer compound ' $\mathrm{A}$ ' ranging from $2.5 \%$ to $20 \%$ (mass ratio). These samples were then mounted on to an aluminium block and tested over a period of two seconds inside the integrating sphere. The luminous flux of the targets was measured when exposed to $1.6 \mathrm{~W}$ of laser power. These results are presented in figure 6 which compares each sample's luminous flux. Clearly the sample containing $10 \%$ phosphor is demonstrating the highest luminous flux. The superiority of the $10 \%$ sample is related to how this particular mix affects the total number of phosphor particles exposed to the laser beam and the efficiency of the beam scattering within the target structure. It can be argued that below this concentration there are not enough phosphor particles activated and above this level the dispersion density of the phosphor particles prevents the scattering of the laser within the target while possibly also preventing light coming out from the target. 


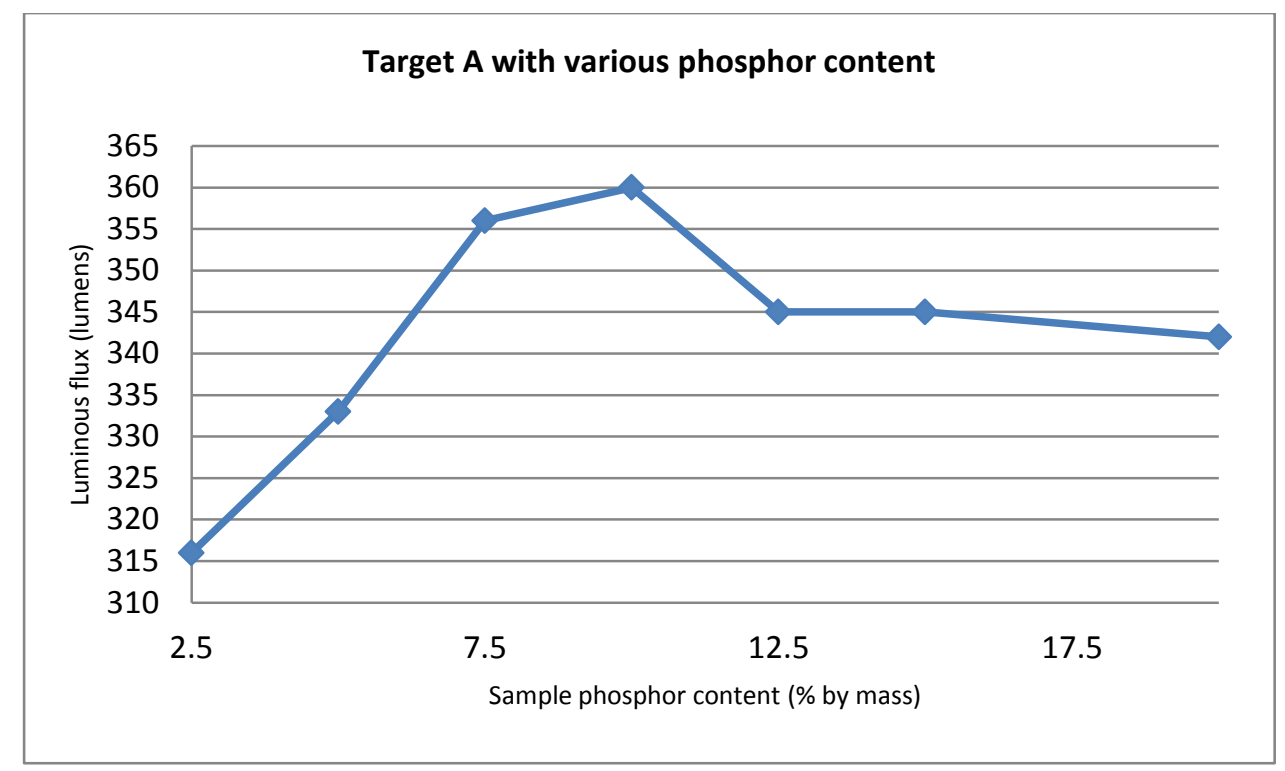

Figure 6. The relationship between phosphor concentration in target A and the Luminous flux.

The A targets containing various different percentages of phosphor mass were then examined for the drop of Luminous flux over time (due to thermal energy). All the A targets exhibited similar behaviour. The data obtained from the A target containing $10 \%$ of the phosphor are presented in figure 7. As all other A targets exhibited similar behaviour in terms of reduction in light output over time, this suggests that the thermal conductivity of the heat transfer compound incorporated into the A targets is the dominant factor in dissipating heat from the target. The superiority of this system over the control system is also demonstrated in figure 7 where the drop in luminous flux is significantly smaller than in Figure 2 with a time to half brightness of approximately 100 seconds.

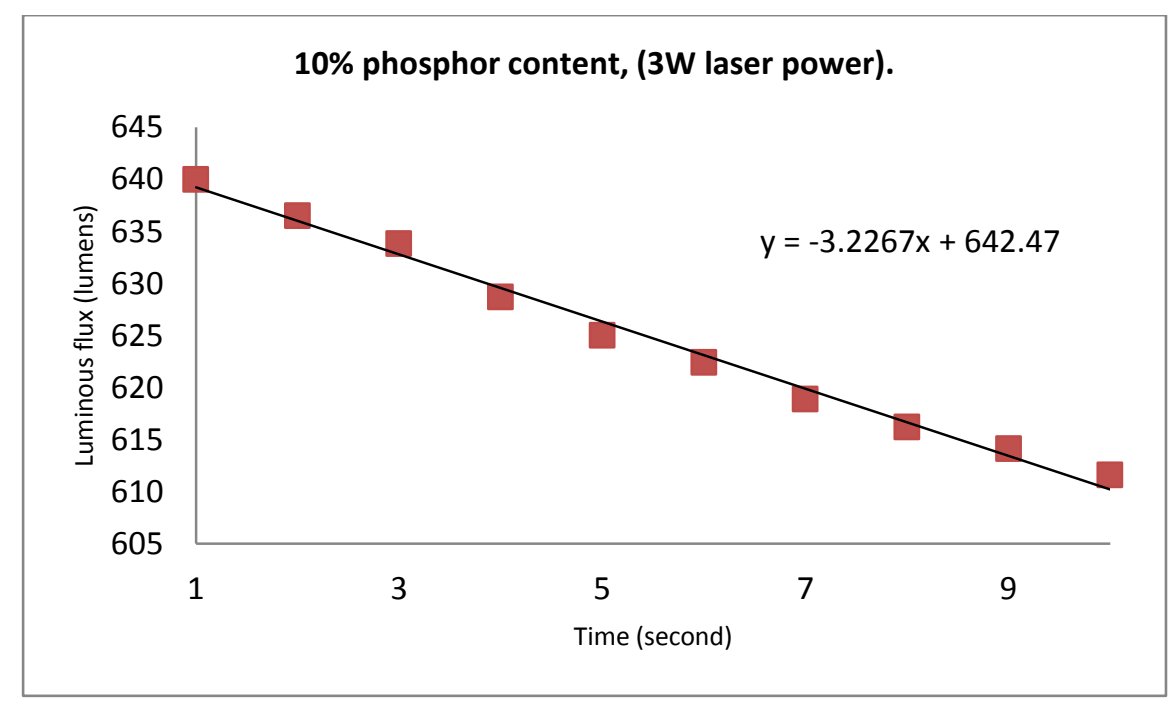

Figure 7. Drop in luminous flux over a period of 10 seconds, with the $10 \%$ phosphor in target A.

The B target samples relied on a different mechanism (applying the phosphor to a heat conducting substrate) to perform the heat dissipation role when the target was exposed to the laser. The experiments were carried out exactly as in the ' $A$ ' samples and were evaluated with a laser power of $2.38 \mathrm{~W}$. However, targets produced with this technique did not suffer the decrease in luminous flux 
seen for the A samples. This facilitated a longer timeframe for the measurements. Hence the experiment was carried out over a 25 hour period and the results are presented in figures 8 . From this Figure it can be seen that after some initial stability issues this system of heat dissipation eliminated the problem observed with the A targets and a time to half brightness of 905 hours is predicted from a linear fit to the linear portion of the data. The luminous flux, chromaticity index and colour temperature of the light produced remain relatively constant during the 25 hour period and these parameters are presented in figures $8 \& 9$. The drawback of this system however is that above the $2.4 \mathrm{~W}$ laser power, the target suffered from catastrophic deterioration. So although it is superior to the ' $\mathrm{A}$ ' target construction, there is a limit to the maximum power that can be used.

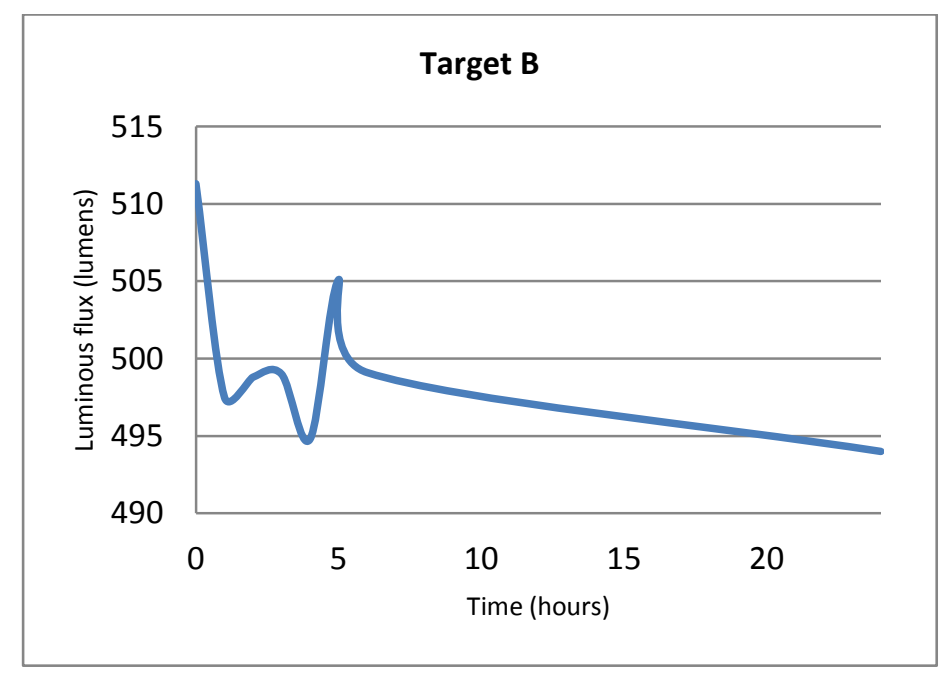

Figure 8. Luminous flux of the target ' $\mathrm{B}$ ' over a period of 25 hours.

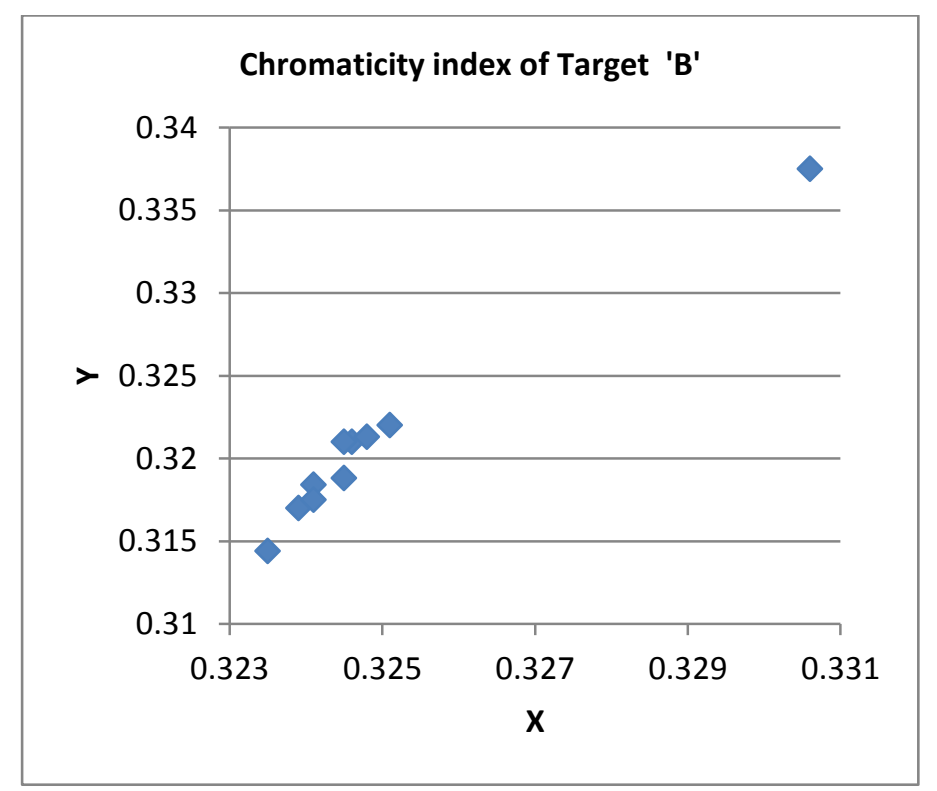

Figure 9. Chromaticity index of the emission from target system 'B' over a period of 25 hours. 
The $\mathrm{C}$ target was constructed as whole module. The module was a compact design made of the laser diode and its heat sink, driver and the phosphor target and its heat sink within one body (figure 1, right). The laser power used in this system was a constant beam of $5 \mathrm{~W}$. The module was placed inside the integrating sphere and the sphere was calibrated so that the amount of light absorbed by the body of the module was taken into account during analysis as presented in figure 10.

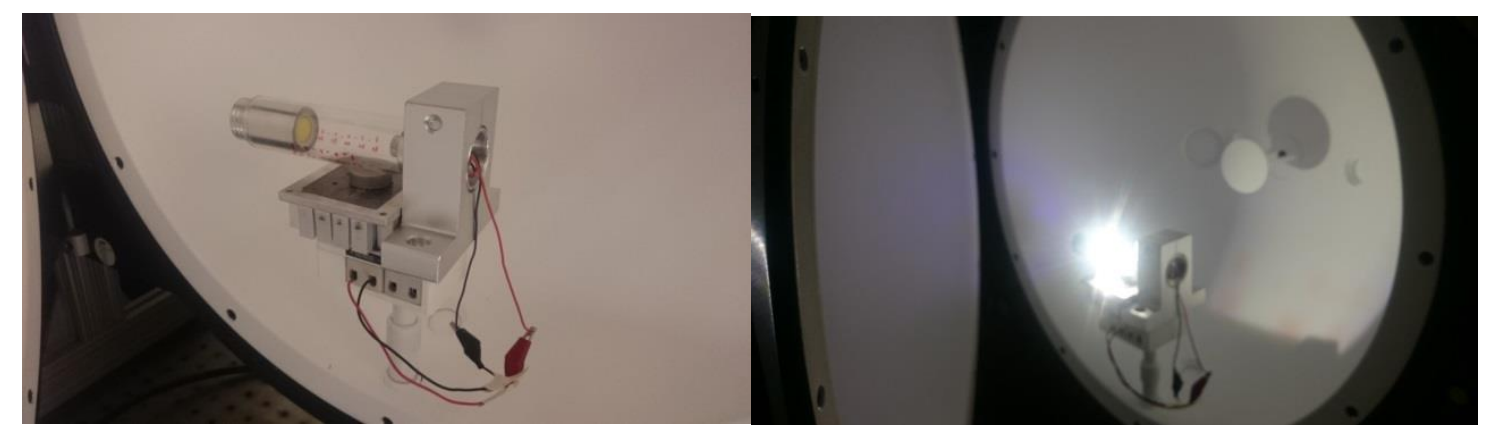

Figure 10. Target system ' $\mathrm{C}$ ' inside the integrating sphere in $\mathrm{ON}$ and OFF mode.

The module in ON and OFF states inside the sphere is presented in figure 10. This module was particularly designed so that it could be fitted into an automobile head lamp. By adjusting the focus of the beam, the luminous flux of the light output could be manipulated. The analysis of the emission from this unit over a period of $20 \mathrm{~min}$ is presented in figure 11 (left). For comparison a control target (with no thermal management) was placed in this module and the loss in emission characteristics is presented in figure 11 (right). Figure 12 presents the data obtained for luminous flux (lumens) of the module over 20 minutes when fitted with a $\mathrm{C}$ type target.

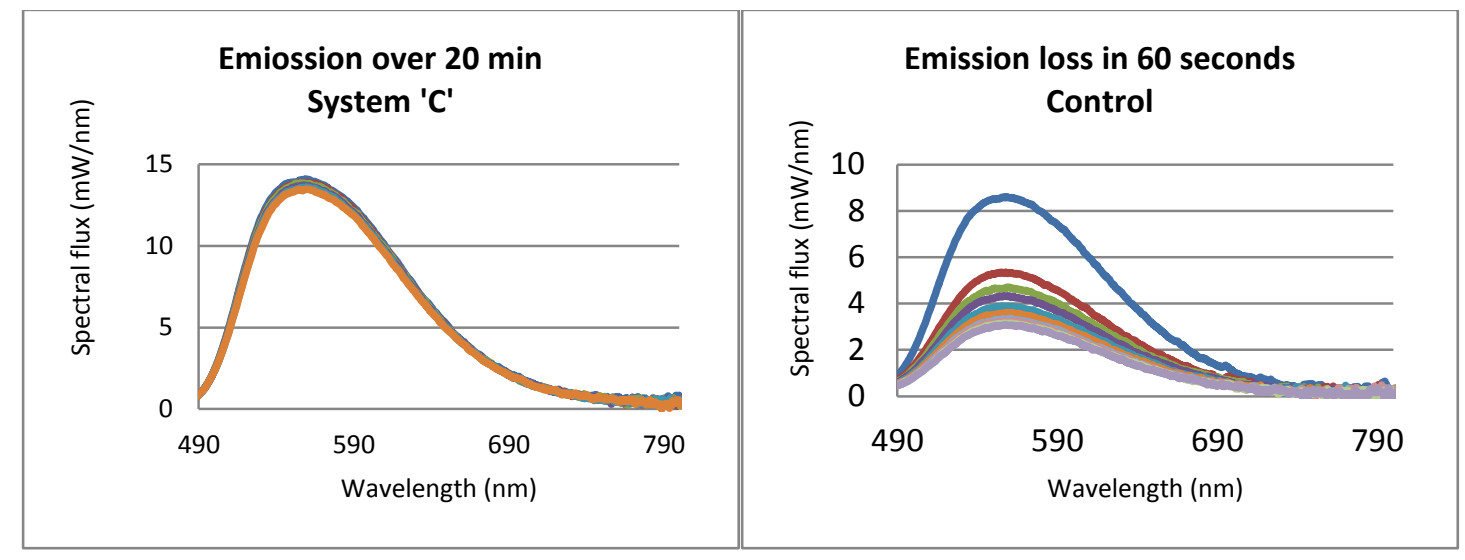

Figure 11. Comparison of emission stability of a $\mathrm{C}$ Target and a control using a $5 \mathrm{~W}$ laser power. The data for the control system were taken every 10 second. 


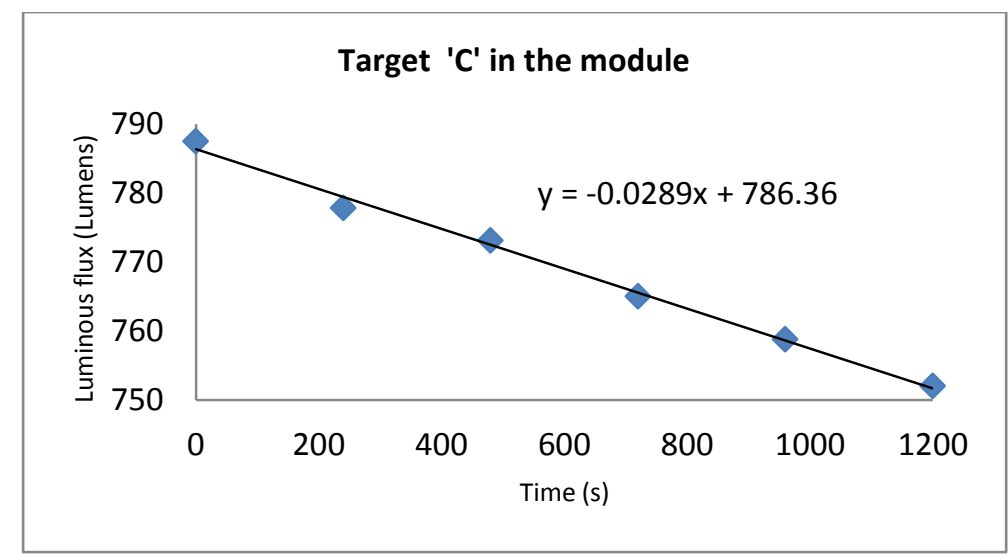

Figure 13. Luminous flux of the target $\mathrm{C}$ over a period of 20 minutes.

The thermal stability of the module containing the $\mathrm{C}$ target was monitored using a thermal camera as described previously. This was carried out by positioning the thermal camera above the module during operation and focusing the camera on the desired location where the target was installed. Figure 14 shows how the experiment was performed, while figure 15 presents the results.

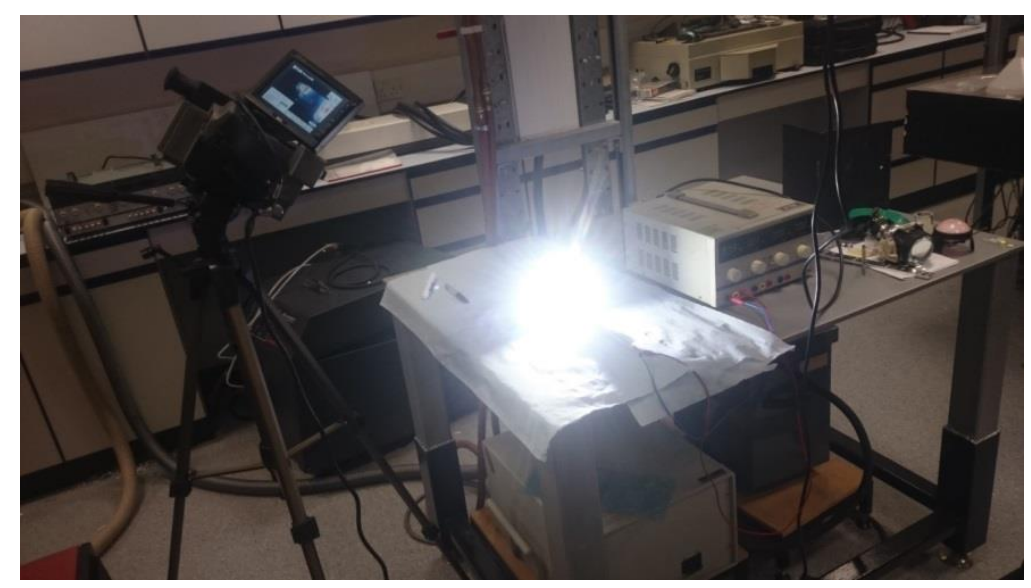

Figure 14. The setup for monitoring the thermal variations within the module target compartment. 

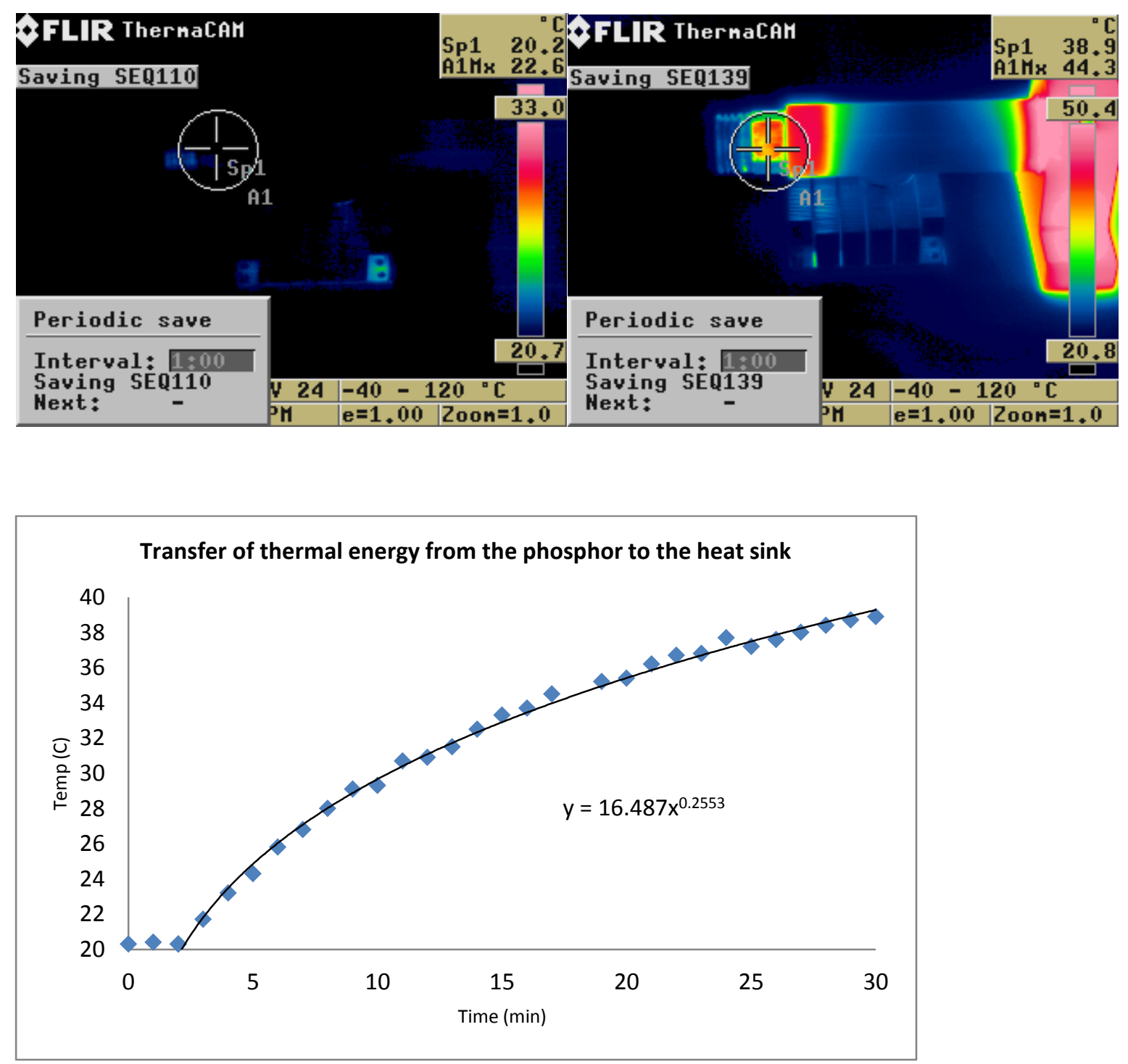

Figure 15. Visual images of thermal monitoring at time 0.0 (Top left) and at time $30 \mathrm{~min}$ (Top right) of the phosphor targets heat sink. The rise in the temperature recorded at 1 minute intervals over the 30 minutes is shown in the graph.

The thermal stability of the $\mathrm{C}$ target was analysed as presented in Figure 15 . The temperature of the $\mathrm{C}$ target heat sink was monitored to demonstrate efficient transfer of the thermal energy from the phosphor target to its heat sink over a period of 30 minutes at 1 minute intervals, whilst the laser was on. As is illustrated in Figure 15 above the $\mathrm{C}$ target is efficiently transferring its thermal load into the heat sink. The phosphor heat sink was isolated from the main laser heat sink so that the rise in its temperature as evidence of heat being transferred from the $\mathrm{C}$ target could easily be monitored.

The Module with target $\mathrm{C}$ mounted was operated for a period of 300 minutes (5 hours) and the stability of the emission and chromaticity were monitored at one hour intervals; the results are presented in figure 16 (a small amount of power instability is observed on this expanded scale). In both cases the properties are seen to be stable over the 5 hour period. 

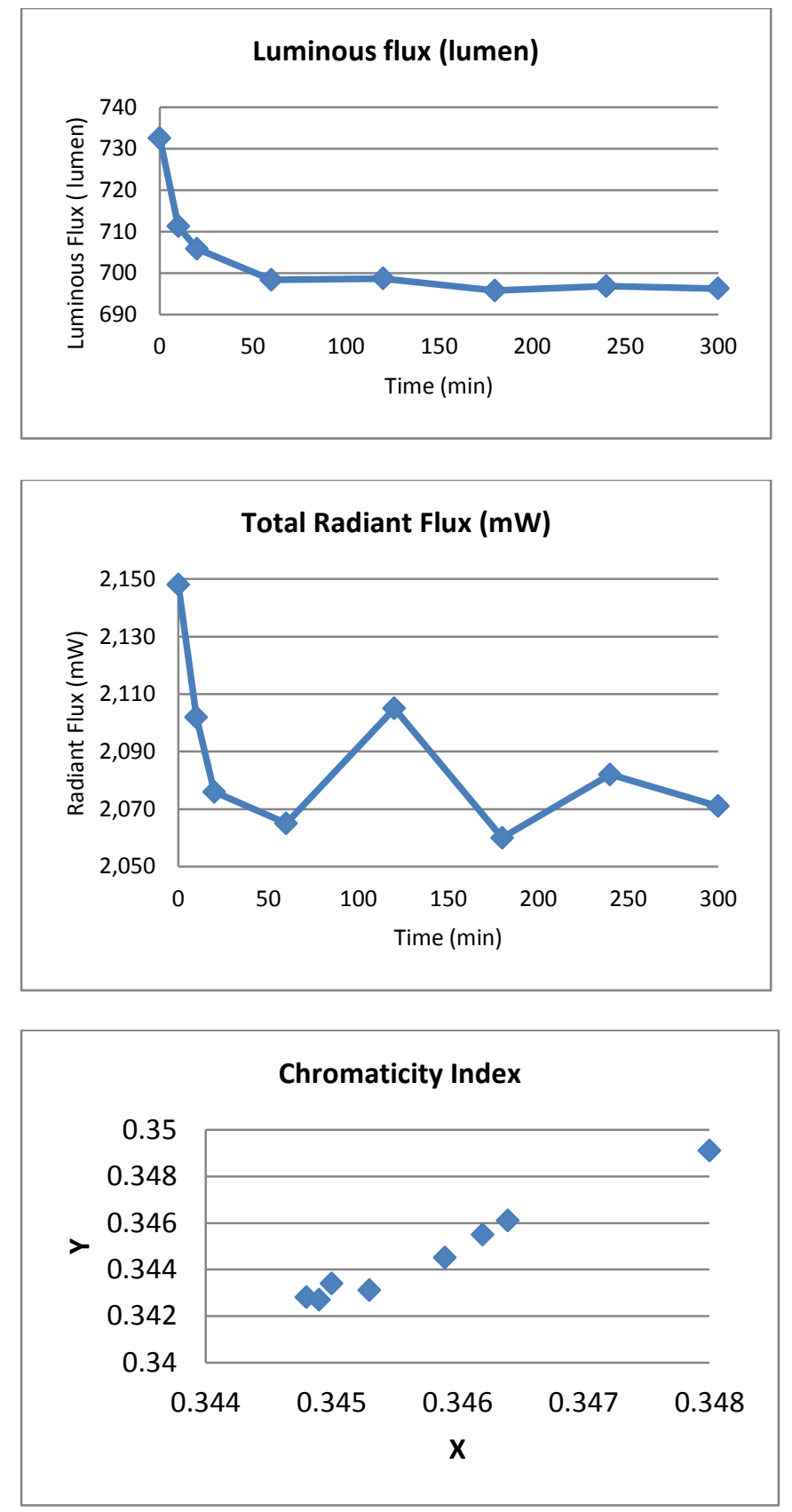

Figure 16. Stability of emission from Target C, under $5 \mathrm{~W}$ laser power over a period of 5 hours.

\section{Conclusion}

Phosphor targets activated with a laser beam can be utilised in a variety of applications which require bright white light source, however the performance of these devices is highly dependent on the thermal management of the target material. The heat generated by the laser beam can be a problem. We have herein demonstrated a solution for this problem by preparing thermally stable phosphor screens. Further enhancement of our heat management and geometry of the final devices will open opportunities in utilising various phosphor combinations to have a high brightness light source with tuneable characteristics dependant on the type of the laser and phosphors used. We have further 
demonstrated herein that high brightness white light can be generated from BLDs and YAG:Ce phosphor and they can be made stable by controlling the removal of the heat generated.

\section{Acknowledgements}

We would like to thank Brunel University London for allocation of funds from EPSRC grant No. EP/K504208/1.

\section{References}

1. S. Nakamura, S. Pearton and G. Fasol "The Blue Laser Diode: The Complete Story" Springer Science \& Business Media, 17 Apr 2013 - Science - 368 pages.

2. Y. Narukawa, I. Niki, K. Izuno, M. Yamada, Y. Murazaki, and T. Mukai, "Phosphorconversion white light emitting diode using InGaN near-ultraviolet chip," Jpn. J. Appl. Phys. 41, L371-L373 (2002).

3. H.Y. Ryu, D.H. Kim, “ High-Brightness Phosphor-conversion white light source using InGaN Blue Laser Diode," J of the Opt Society of Korea. Vol 14, No 4 (2010).

4. Y. Xu, L. Chen, Y. Li, G. Song, and Y. Wang, "Phosphor-conversion white light using InGaN ultraviolet laser diode," Appl. Phys. Lett. 92, 021129 (2008).

5. T. Kozaki, S. Nagahama, and T. Mukai, "Recent progress of high-power GaN-based laser diodes," Proc. SPIE 6485, 648503 (2007). 